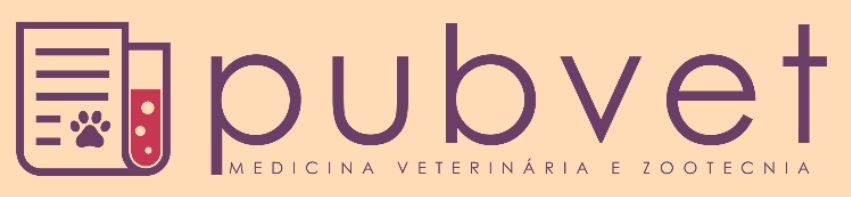

https://doi.org/10.31533/pubvet.v13n8a402.1-7

\title{
Hipospadia anal em um canino: relato de caso
}

\author{
Marcio Fernando Weber Brito ${ }^{*} \bullet$, Charles Silva de Lima ${ }^{2} \bullet$, Vanessa Milech ${ }^{3} \bullet$, Felipe Rosa

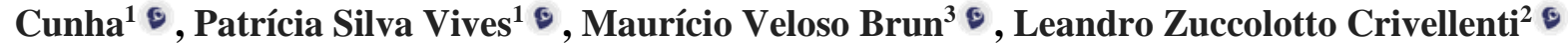 \\ ${ }^{I}$ Universidade Federal de Pelotas, Faculdade de Veterinária, Departamento de Clínica Veterinárias, Pelotas - RS, Brasil. \\ ${ }^{2}$ Universidade de Franca; Faculdade de Veterinária, Departamento de Clínica e Cirurgia Veterinária; Franca - SP, Brasil. \\ ${ }^{3}$ Universidade Federal de Santa Maria, Curso de Medicina Veterinária, Departamento de Clínica e Cirurgia de Pequenos Animais; Santa Maria - RS, Brasil. \\ *Autor para correspondência, e-mail: xmarciobrito@hotmail.com
}

Resumo. O objetivo deste trabalho é relatar um caso de hipospadia anal em um canino macho de dois meses de idade, abordando diagnóstico e conduta terapêutica. O paciente foi atendido no Hospital de Clínicas Veterinárias da Universidade Federal de Pelotas, onde foram relatadas alterações no ato de defecação e, principalmente, micção, sendo ambos realizados pelo ânus. Procederam-se exames hematológicos e de urinálise, além de exame ultrassonográfico abdominal, onde as imagens de arquitetura renal, vesical e intestinal apresentavam significativas alterações. Após a estabilização das debilidades clínicas o paciente foi encaminhado para correção da hipospadia, penectomia e criptorquidectomia. Observou-se que a conduta clínica e cirúrgica logrou êxito, visto a melhora do quadro e ausência de sinais clínicos posteriores.

Palavras chave: anomalidade congênita, canino, cirurgia, uretra

\section{Anal hypospadias in a dog: case report}

Abstract. The aim of this study was to report a case of anal hypospadias in a male canine, and also the diagnosis and therapeutic management. The patient was treated at the Veterinary Hospital of Federal University of Pelotas. Alterations were observed in both defecation and, especially micturition that was eliminated by the anus. CBC, urinalysis and abdominal ultrasound was performed. Abnormalities were detected in kidneys, bladder and intestine. After clinical stabilization the patient was referred for hypospadias correction, penectomy and cryptorchidectomy. Clinical and surgical procedure was adequate, and improvement on score condition was observed.

Keywords: congenital abnormalities, canine, surgery, urethra

\section{Hipospadia anal en canino: relato de caso}

Resumen. El objetivo de este trabajo es relatar un caso de hipospadia anal en un canino macho de dos meses de edad, abordando diagnóstico y conducta terapéutica. El paciente fue atendido en el Hospital de Clínicas Veterinarias de la Universidad Federal de Pelotas, donde se relataron alteraciones en el acto de defecación y, principalmente, micción, siendo ambos realizados por el ano. Se realizaron exámenes hematológicos y de urinálisis, además de examen ultrasonográfico abdominal, donde las imágenes de arquitectura renal, vesical e intestinal presentaban significativas alteraciones. Después de la estabilización de las debilidades clínicas el paciente fue encaminado para corrección de la hipospadia, penectomía y criptorquidectomía. Se observó que la conducta clínica y quirúrgica logró éxito, visto la mejora del cuadro y ausencia de signos clínicos posteriores.

Palabras clave: anomalía congénita, canino, cirugía, uretra 


\section{Introdução}

A hipospadia é uma malformação congênita na qual a uretra cessa em porção ventral e caudalmente à sua localização normal (Memon \& Mickelsen, 2004), sendo causada por uma combinação de fatores genéticos e ambientais (Switonski et al., 2018). De acordo com a localização da abertura uretral pode ser classificada como glandular, peniana, escrotal, perineal e anal (MacPhail, 2014; Switonski et al., 2012), ocorrendo tanto em machos quanto em fêmeas (Cashmore \& Ladlow, 2010; Memon \& Mickelsen, 2004). Especialmente nos machos, esta enfermidade pode estar relacionada com a falha da fusão do prepúcio e ausência ou subdesenvolvimento do pênis (Slatter, 2007), bem como, criptorquidia (Switonski et al., 2018).

O diagnóstico dá-se por meio da observação da anormalidade da uretra peniana, pênis, prepúcio e/ou escroto (MacPhail, 2014), dentre a raças descritas com maior frequência citam-se o cocker spaniel, collie, doberman pinscher, dinamarquês, boston terrier e pastor alemão (Gobello et al., 2003; Switonski et al., 2018). Os sinais clínicos possíveis são incontinência urinária, piodermas perineais por contato de urina e infecções do trato urinário (ITU) (Matthews, 2008). Na maioria dos casos aconselha-se correção cirúrgica, para idades superiores há dois meses, objetivando estética e funcionalidade da genitália, realizando-se correção prepucial, uretral ou penectomia (Angeli et al., 2007; MacPhail, 2014).

O objetivo deste trabalho é relatar um caso de hipospadia em um canino, abordando descrição do caso, diagnóstico e terapia preconizada.

\section{Relato de caso}

Atendeu-se um paciente canino, macho, sem raça definida, de dois meses de idade, pesando $2,9 \mathrm{~kg}$. De acordo com relato do tutor este teria sido o único, em uma ninhada de oito filhotes, que havia nascido sem cauda e teria uma alteração morfológica no prepúcio. Ainda, relatou que o canino apresentava tenesmo fecal e aparentemente "urinava pelo ânus", manifestando sensibilidade dolorosa. Ao exame físico geral o paciente apresentava-se magro (score 4 de 9); porém, hidratado, alerta, com mucosas normocoradas e sem alteração em parâmetros vitais. Ao avaliar-se a genitália externa pode-se observar que o prepúcio possuía abertura ventral, expondo a mucosa peniana. O pênis era subdesenvolvido e de aspecto atrofiado (Figura 1) e os testículos encontravam-se em região inguinal. Além disso, a região perineal apresentava-se eritematosa, alopécica e com vestígios fecais de aspecto diarreico. Também foi constatada a ausência de vértebras coccígeas.

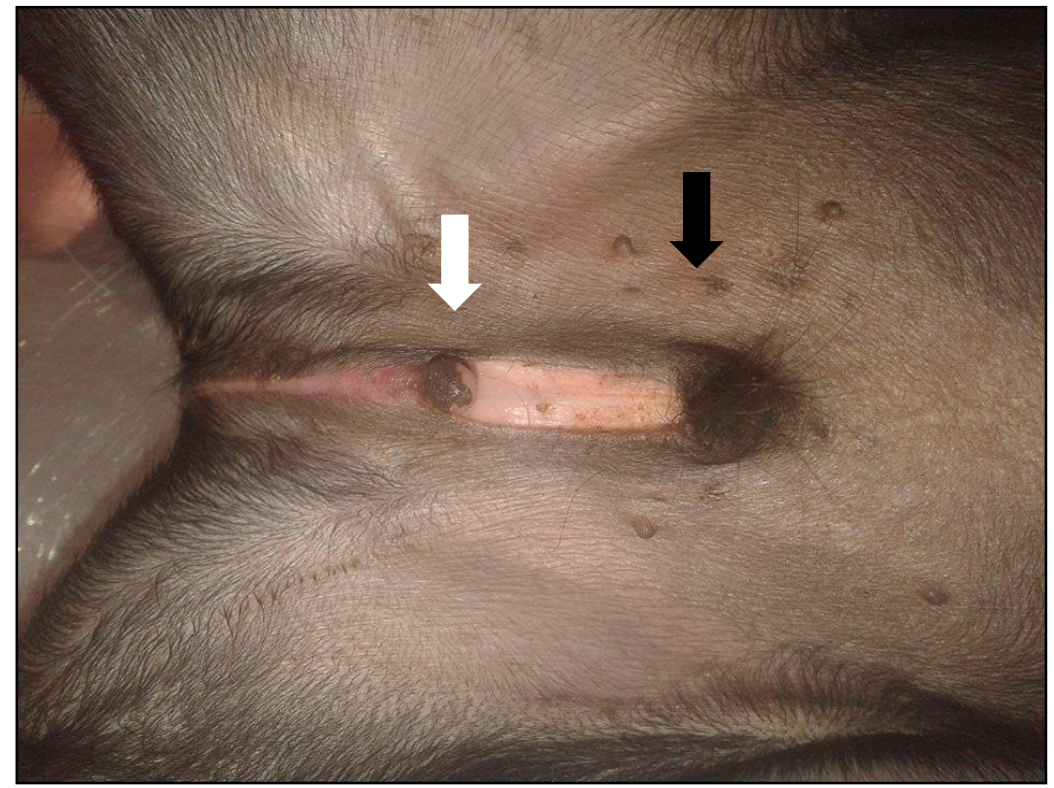

Figura 1. Vista ventral da região abdominal caudal evidenciando a genitália externa. O prepúcio apresentava abertura ventral expondo a mucosa peniana (seta preta). O pênis era subdesenvolvido e de aspecto atrofiado (seta branca). 
Procederam-se a coleta de amostras sanguíneas para realização de hemograma e dosagem sérica de alanina aminotransferase (ALT), fosfatase alcalina (FA), albumina, ureia e creatinina, além da solicitação de urinálise. Ainda, o paciente foi encaminhado para realização de exame ultrassonográfico abdominal com ênfase em aparelho geniturinário.

$\mathrm{O}$ resultado do hemograma demonstrou anemia normocítica normocrômica, e eosinofilia. Na avaliação bioquímica sanguínea foi observada leve diminuição da ureia, mas ALT, FA e creatinina apresentavam-se dentro dos valores normais para a espécie, ainda, observou-se discreta hipoproteinemia e hipoalbuminemia (Tabela 1).

Tabela 1. Exames complementares laboratoriais do paciente canino, SRD, macho de 2 meses com hipospadia

\begin{tabular}{lcc}
\hline Exame & Resultado & Valor de Referência \\
\hline Alanina aminotransferase (ALT) & $29 \mathrm{U} / \mathrm{L}$ & $21-102 \mathrm{U} / \mathrm{L}$ \\
Fosfatase Alcalina (FA) & $51 \mathrm{U} / \mathrm{L}$ & $20-150 \mathrm{U} / \mathrm{L}$ \\
Creatinina & $0,4 \mathrm{mg} / \mathrm{dL}$ & $0,5-1,5 \mathrm{mg} / \mathrm{dL}$ \\
Uréia & $14,5 \mathrm{mg} / \mathrm{dL}$ & $21,4-60 \mathrm{mg} / \mathrm{dL}$ \\
Proteínas Plasmáticas Totais & $5,4 \mathrm{~g} / \mathrm{dL}$ & $6-8 \mathrm{~g} / \mathrm{dL}$ \\
Albumina & $2,5 \mathrm{~g} / \mathrm{dL}$ & $2,6-3,3 \mathrm{~g} / \mathrm{dL}$ \\
\hline
\end{tabular}

O estudo ultrassonográfico apontou presença de segmento de alça apresentando paredes finas, com perda de definição das camadas e distendidas por conteúdo fluido (Figura 2). O rim esquerdo com formato anatômico alterado, dimensões preservadas, relação córtico-medular alterada com junção pouco definida, contorno irregular e pelve dilatada (Figura 3). O rim direito aumentado de tamanho $(5,08 \mathrm{~cm}$, ao corte longitudinal) com junção córtico-medular preservada, porém demonstrando pontos hiperecogênicos, não formadores de sombra acústica posterior, com presença de sinal da medular. Ademais, baço apresentava padrão de reatividade, além de linfonodos abdominais aumentados de volume, com contorno regular e parênquima homogêneo e hiperecogênico em relação aos tecidos adjacentes.

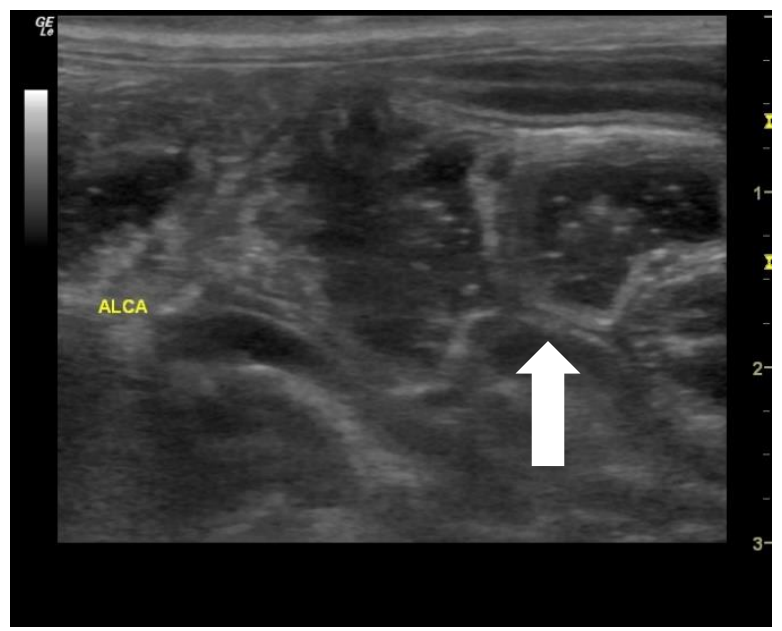

Figura 2. Imagem ultrassonográfica em segmento de alça intestinal apresentando paredes finas (seta), com perda de definição das camadas e distendidas por conteúdo fluido.

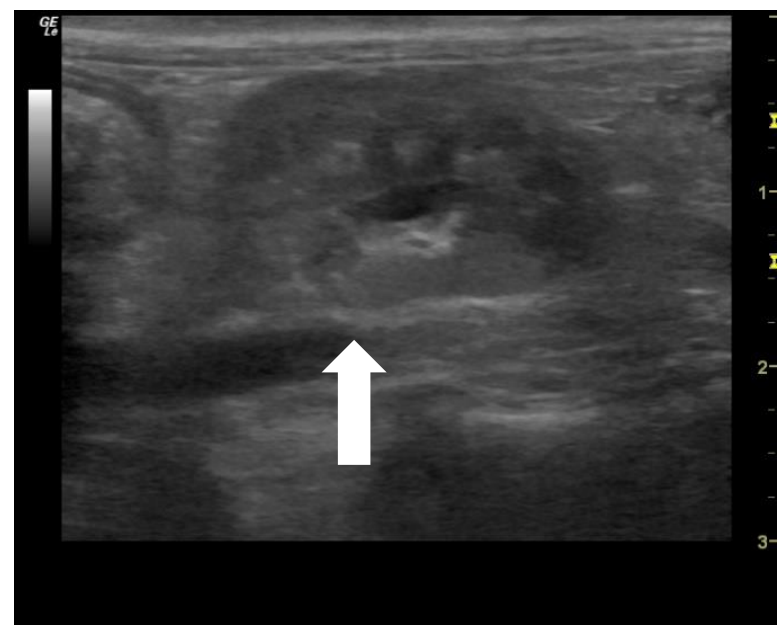

Figura 3. Imagem ultrassonográfica do rim esquerdo com formato anatômico alterado, diminuído, com junção córtico-medular pouco definida, contorno irregular e pelve dilatada (seta).

Durante o período pré-operatório, foi instituído tratamento sintomático. Inicialmente com a instituição de dieta terapêutica (ração intestinal), no sentido de ajustar distúrbio intestinal presente, compensação de má digestão em benefício do estado geral do período de convalescença pré-cirúrgica, além de terapia no sentido de favorecer o ato defecatório. Além disso, estabeleceu-se terapêutica antiálgica e antiespasmódica (dipirona monoidratada $25 \mathrm{mg} / \mathrm{kg}$, a cada 8 horas), antifisética (dimeticona $40 \mathrm{mg} / \mathrm{animal}$, a cada 8 horas) e antimicrobiana (sulfadiazina associado ao trimetoprim, na dose de 30 $\mathrm{mg} / \mathrm{kg}$, a cada 12 horas). 
Para a correção da hipospadia foi realizada a uretrostomia, além de penectomia e criptorquidectomia. Procedeu-se primeiramente com a criptorquidectomia; sendo utilizada a técnica já consolidada conforme descrito por MacPhail (2014). Na sequência, efetuaram-se as técnicas de penectomia total e uretrostomia.

Iniciou-se com uma incisão elíptica ao redor do pênis, seguida da divulsão do pênis e prepúcio caudalmente, até o local da uretrostomia perineal. Foi realizado o reparo da uretra, com o auxílio de uma sonda uretral $\mathrm{n}^{\circ} .8$, e uma incisão paralela à junção mucocutânea em ambos os lados da uretra, que se encontrava espalmada, unindo-se essas bordas sobre a sonda com pontos isolados simples, por meio de fio náilon monofilamentar 4-0, formando-se um túnel uretral até a chegada ao períneo, separando-o da abertura anal. Para a síntese de subcutâneo e pele foram utilizadas respectivamente, padrões de sutura contínuo simples e interrompido simples, com fio absorvível 3-0 no subcutâneo e náilon monofilamentar 4-0 na pele.

No pós-operatório o paciente permaneceu hospitalizado durante três dias para analgesia e observação, além de permanecer com sonda uretral por cinco dias. Notou-se marcada polaciúria e disúria durante um breve período de 48 horas. Passados cinco dias de pós-operatório e remoção da sonda uretral, a micção espontânea deu-se sem intercorrências, através de orifício específico cirurgicamente elaborado (Figura 4A), e o paciente obteve incremento de peso e escore corporal, apresentando estado de consciência alerta, com apetite normal e em ato de normoquesia. Decorridos 10 dias da cirurgia foram retiradas as suturas (Figura 4B) e solicitaram-se periódicos retornos para acompanhamento pediátrico e avaliação de trato urinário. O paciente teve acompanhamento de pós-operatório de aproximadamente seis meses, sendo que neste período não apresentou quaisquer alterações.

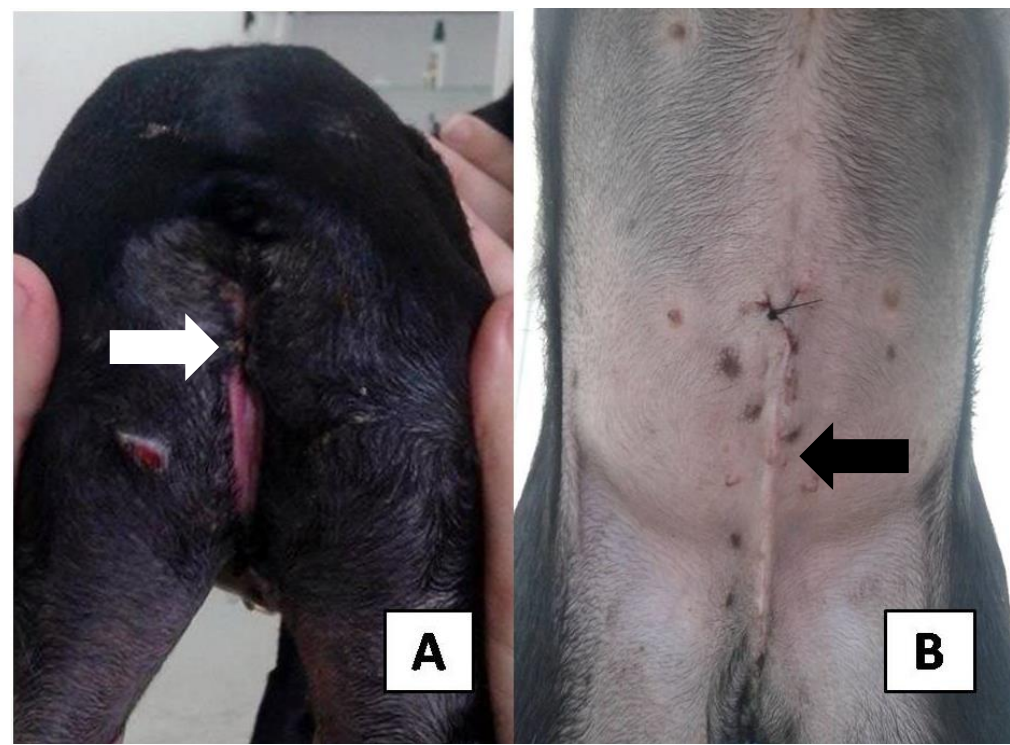

Figura 4. Vista caudal demonstrando área cirúrgica com a formação do óstio uretral (seta branca). Vista ventral da região abdominal caudal evidenciando ferida cirúrgica da correção de hipospadia e penectomia (seta preta)

\section{Discussão}

As alterações hematológicas justificam-se pelo déficit nutricional que o paciente se encontrava no momento da consulta. Situação que ganhou atenção no sentido de propor ajustes nutricionais terapêuticos, levando-o a melhorar estado geral. A eosinofilia poderia sugerir infestação parasitária, visto que o paciente não estava adequadamente desverminado (Sattasathuchana \& Steiner, 2014).

Os achados ultrassonográficos apontaram alterações que sugeriram necessidade imperativa de reavaliações rotineiras dos pacientes. Embora o diagnóstico da hipospadia e visibilização das alterações anatômicas seja externo; portanto, identificadas a olho nu, já foi determinado, em humanos, que exames ultrassonográficos pré-natais podem ser úteis no diagnóstico fetal da hipostadia, ou seja, com valor preditivo de alterações penianas de $84 \%$ e especificamente hipospadia de $72 \%$ (Epelboym et al., 2017). 
Sabe-se que cães portadores de hipospadia podem ser assintomáticos ou apresentar sinais clínicos como incontinência urinária, dermatite perineal e/ou infecções urinárias recorrentes (Adelsberger \& Smeak, 2009; MacPhail, 2014). Corroborando com o supramencionado, o paciente relatado apresentava lesão dermatológica em região perineal por contato constante de fezes e urina, além de também demonstrar infecção urinária, possivelmente originada por contaminação ascendente de microrganismos em vias urinárias. Embora alguns autores (MacPhail, 2014; Meyers-Wallen, 2012; Poth et al., 2010) considerem esta uma enfermidade incomum em caninos; Switonski et al. (2018) supõe que a hipospadia não é rara, mas que sua incidência é subestimada, devido ao fato de que o exame clínico do pênis e a localização do orifício uretral em cães não são rotineiramente executados. Na experiência destes autores, a hipospadia tem sido uma enfermidade de pouca casuística, sugerindo assim a adequada avaliação dos pacientes desde a primeira consulta pediátrica.

A malformação da uretra peniana, do pênis, do prepúcio e do escroto está ligeiramente associada à deficiência de testosterona durante a morfogênese, como já citado por outros autores (Memon \& Mickelsen, 2004). Também está demonstrado que a enfermidade pode estar relacionada com outras malformações congênitas (Valente et al., 2014) e, além de hipospadia, o paciente relatado apresentava ausência de vertebras coccígeas. No mesmo relato supracitado (Guimarães et al., 2013; Valente et al., 2014) são trazidos dois casos envolvendo cães machos, diagnosticados com hipospadia e completa ausência de vértebras coccígeas presumindo, assim, que possa haver uma associação entre estes achados. Entretanto, em estudo retrospectivo sobre hipospadias (Switonski et al., 2018), tal distúrbio não é mencionado. No respectivo caso, a hipospadia diagnosticada foi classificada como anal, uma vez que o meato uretral se localizava juntamente ao ânus (MacPhail, 2014). Esta é uma observação interessante ao considerarmos que a maioria das hipospadias tem localização peniana e perineal (Switonski et al., 2018; Torres et al., 2007). Desta forma, este relato também traz uma abordagem cirúrgica que pode ser aplicada em casos de hipospadia anal.

O posicionamento ectópico da abertura da uretra, na maioria dos casos, requer cirurgias reconstrutivas (Lyle, 2003), principalmente ao ponderar-se que podem existir vários graus de tensão uretral e deficiência do corpo esponjoso que, frequentemente, são acompanhados por falha de fusão do prepúcio e subdesenvolvimento do pênis (Cashmore \& Ladlow, 2010). Por isso, após duas semanas de acompanhamento e estabilização, o paciente foi submetido ao procedimento cirúrgico de correção de hipospadia, penectomia e criptorquidectomia. A uretrostomia perineal ou escrotal combinada com a remoção de prepúcio e vestígios de tecido peniano visam correção funcional e também a estética do paciente (Guimarães et al., 2013; Valente et al., 2014) e, no presente caso, proporcionaram excelentes resultados terapêuticos. Ainda, cabe ressaltar que poucos são os relatos de correção cirúrgica de hipospadia, se comparar os dados veterinários aos humanos. As técnicas relatadas abordam principalmente casos em que o meato uretral está localizado distalmente ao escroto, objetivando corrigir efeitos deletérios como acúmulo de urina dentro do prepúcio ou exposição do pênis, embora técnicas recomendadas para correção de hipospadias graves requerem a combinação de penectomia e uretrostomia (Cashmore \& Ladlow, 2010). A criptorquidectomia é de suma importância, inicialmente porque estes testículos são mais suscetíveis às torções ou neoplasias (Guimarães et al., 2013; Valente et al., 2014), mas também, por não recomendarem-se os animais afetados para a reprodução (MacPhail, 2014) considerando, neste último, não só a criptorquidia, mas também a hipospadia.

Cashmore \& Ladlow (2010) descrevem uma técnica reconstrutiva para correção de hipospadia grave em canino de seis semanas, onde se realizou a criação de um conduto uretral empregando retalho prepucial no intuito de transferir o meato uretral da região perineal para a inguinal. Tal manobra visa evitar a uretrostomia perineal, menos indicada por alguns autores (MacPhail, 2014) por ser frequentemente associada a maior contaminação do meato uretral e consequentemente ocorrência de ITU. Apesar deste fato, a uretrostomia perineal realizada no paciente em questão não demonstrou qualquer alteração pós-operatória, enfatizando que esta pode ser aplicada em casos de hipospadia anal. Compreendendo que a hipospadia trata-se de uma alteração congênita que pode ocorrer como um distúrbio isolado, ou em combinação com outras alterações no sistema urogenital (Bouty et al., 2016), fica clara a necessidade de terapêutica baseada na correção cirúrgica de tal enfermidade. Ainda, neste caso a conduta terapêutica cirúrgica também foi eficaz no controle das infecções ascendentes de trato urinário do paciente, concordando com relatos de literatura (Cashmore \& Ladlow, 2010; Valente et al., 
2014) que consideraram o auxílio da cirurgia no controle de infecções urinária pela separação anal e uretral em casos de fístulas uretroretais e hipospadia perianal proximal.

\section{Conclusão}

O presente relato reforça a importância desse tipo de abordagem em pacientes com hipospadia, visto que, ainda são escassos os relatos na literatura veterinária. O diagnóstico precoce da anormalidade, a estabilização clínica do paciente e o procedimento cirúrgico realizado, possibilitaram eliminar os sinais clínicos e permitiram melhor qualidade de vida ao paciente.

\section{Referências bibliográficas}

Adelsberger, M. E. \& Smeak, D. D. (2009). Repair of extensive perineal hypospadias in a Boston terrier using tubularized incised plate urethroplasty. The Canadian Veterinary Journal, 50(9):937-942.

Angeli, A. L., Rocha, T. M. M., Maia, R., Alcântara, M. A., Frehse, M. \& Tanaka, N. M. (2007). Hipospadia perineal em cão da raça buldogue inglês: primeiro relato. Acta Scientiae Veterinariae, 35591-592.

Bouty, A., Lefevre, Y., Harper, L. \& Dobremez, E. (2016). Urethral duplication in girls: Three cases associating an accessory epispadiac urethra and a main hypospadiac urethra. Journal of Pediatric Urology, 12(4):209. e201-209. e205.

Cashmore, R. G. \& Ladlow, J. F. (2010). Creation of a urethral conduit from a preputial indirect flap in a dog with perineal hypospadias. Veterinary Surgery, 39(1):14-20.

Epelboym, Y., Estrada, C. \& Estroff, J. (2017). Ultrasound diagnosis of fetal hypospadias: Accuracy and outcomes. Journal of Pediatric Urology, 13(5):484. e481-484. e484.

Gobello, C., De Luca, J. C., Corrada, Y. A., García, P. P. \& García, M. (2003). Penile hypoplasia in a rottweiler: a case report. Analecta Veterinaria, 23(1):38-41.

Guimarães, L. D., Bourguignon, E., Santos, L. C., Duarte, T. S., Andrade, E. C. \& Borges, A. P. B. (2013). Canine perineal hypospadias. Arquivo Brasileiro de Medicina Veterinaria e Zootecnia, 65(6): 1647-1650.

Lyle, S. K. (2003). Disorders of sexual development. In M. V. R. Kustritz (Ed.), Small animal theriogenology (pp. 1-31). USA: Elsevier Science.

MacPhail, C. M. (2014). Cirurgia dos sistemas reprodutivo e genital. In T. W. Fossum (Ed.), Cirurgia de pequenos animais. Rio de Janeiro, Brasil: Elsevier.

Matthews, H. K. (2008). Doenças da Uretra. In S. J. Birchard \& R. G. Sherding (Eds.), Manual Saunders de clínica de pequenos animais (pp. 943-950). São Paulo, São Paulo, Brasil: Editora Roca.

Memon, M. A. \& Mickelsen, W. D. (2004). Distúrbios hereditários e congênitos dos sistemas reprodutivos do macho e da fêmea. In S. J. Ettinger \& E. C. Feldman (Eds.), Tratado de medicina interna veterinária doenças do cão e do gato. (pp. 1668-1669). São Paulo, São Paulo, Brasil: Guanabara Koogan.

Meyers-Wallen, V. (2012). Gonadal and sex differentiation abnormalities of dogs and cats. Sexual Development, 6(1-3):46-60.

Poth, T., Breuer, W., Walter, B., Hecht, W. \& Hermanns, W. (2010). Disorders of sex development in the dog-Adoption of a new nomenclature and reclassification of reported cases. Animal Reproduction Science, 121(3-4):197-207.

Sattasathuchana, P. \& Steiner, J. (2014). Canine eosinophilic gastrointestinal disorders. Animal Health Research Reviews, 15(1):76-86.

Slatter, D. H. (2007). Manual de cirurgia de pequenos animais. São Paulo: Manole.

Switonski, M., Dzimira, S., Aleksiewicz, R., Szczerbal, I., Nowacka-Woszuk, J., Krzeminska, P., . . . Nizanski, W. (2018). Hypospadias is not rare in dogs: five new cases, a retrospective study, and a review of the literature. Sexual Development, 12(5):244-250.

Switonski, M., Payan-Carreira, R., Bartz, M., Nowacka-Woszuk, J., Szczerbal, I., Colaco, B., . . . Nizanski, W. (2012). Hypospadias in a male (78, XY; SRY-positive) dog and sex reversal female 
(78, XX; SRY-negative) dogs: clinical, histological and genetic studies. Sexual Development, 6(13):128-134.

Torres, J., Sato, A. \& Torres, O. (2007). Hipospadia severa con defecto concurrente del pene, escroto y prepucio en canino. REDVET. Revista Electrónica de Veterinaria, 8(3):1-3.

Valente, F. S., Gonzalez, P. C. \& Contesini, E. A. (2014). Hipospadia perineal em um cão: relato de caso. Arquivo Brasileiro de Medicina Veterinaria e Zootecnia, 66(3):757-762.

Recebido: 29 de maio, 2019.

Aprovado: 29 de junho, 2019.

Publicado: 10 de outubro, 2019.

Licenciamento: Este artigo é publicado na modalidade Acesso Aberto sob a licença Creative Commons Atribuição 4.0 (CC-BY 4.0), a qual permite uso irrestrito, distribuição, reprodução em qualquer meio, desde que o autor e a fonte sejam devidamente creditados 